Article original

\title{
Localisation des centres de radiothérapie: une analyse géographique exploratoire pour la Belgique ${ }^{\text {ir }}$
}

\author{
Location of radiotherapy centers: An exploratory geographic analysis for Belgium \\ C. Cotteels ${ }^{a}$, D. Peeters ${ }^{\mathrm{a}, \mathrm{b}}$, P.A. Coucke ${ }^{\mathrm{c}}$, I. Thomas $^{\mathrm{a}, *, \mathrm{~b}, \mathrm{~d}}$ \\ ${ }^{a}$ Center for Operations Research and Econometrics, université catholique de Louvain, voie du Roman-Pays 34, boîte L1.03.01, 1348 Louvain-la-Neuve, Belgique \\ ' École de géographie, université catholique de Louvain, Louvain-la-Neuve, Belgique \\ ' Service radiothérapie-oncologie, $\mathrm{CHU}$, université de Liège, Liège, Belgique \\ ${ }^{\mathrm{d}}$ Centre de recherches F.R.S., F.N.R.S., Bruxelles, Belgique
}

\section{N F O A R T I C L E}

\section{Historique de l'article :}

Reçu le 6 janvier 2012

Reçu sous la forme révisée

le 18 mai 2012

Accepté le 4 juillet 2012

\section{Mots clés :}

Radiothérapie

p-médiane

Modèle localisation-affectation

Géographie

\section{R É S U M É}

Objectif de l'étude. - La distance entre le domicile du patient et le centre de radiothérapie peut constituer un frein à la compliance du patient au traitement, voire au choix du traitement. C'est pourquoi il convient de réfléchir à la localisation des centres par rapport à la répartition géographique de la demande en prenant en compte le coût du déplacement. L'objectif de cette étude était double: d'abord, cartographier les localisations actuelles des centres de radiothérapie à l'échelle d'un pays et ensuite, évaluer les disparités spatiales observées par des outils appropriés.

Matériels et méthode. - Un modèle de recherche opérationnelle ( $p$-médiane) a été utilisé pour suggérer les localisations et affectations optimales et les comparer aux actuelles. Il s'agissait certes d'une étude exploratoire avec des entrants (inputs) simples, mais ils aidaient à mieux comprendre la géographie actuelle de l'offre en Belgique ainsi que ses éventuelles inadéquations.

Résultats-conclusion. - Il ressort que la situation actuelle est en moyenne acceptable en termes d'accessibilité au service et que l'outil utilisé présente des potentialités énormes en matière d'aide à la décision afin de tendre à la fois vers un système spatial équitable et efficace.

(c) 2012 Société française de radiothérapie oncologique (SFRO). Publié par Elsevier Masson SAS. Tous droits réservés.

\section{A B S T R A C T}

Purpose. - The distance between the patient's home and a radiotherapy department may represent a hurdle for the patient and influence treatment choice. Therefore, it is necessary to check whether the geographical distribution of radiotherapy centers is in accordance with cancer incidence, taking also into account the cost of travelling to the radiotherapy department. The objective of this study is double; first, to map the current locations of radiotherapy centers across the country and second, to evaluate the observed spatial disparities with appropriate tools.

Materials and methods. - A model of operational research ( $P$-median) is used to suggest the optimal locations and allocations and to compare them with the current situation. This is an exploratory study with simple inputs. It helps to better understand the current geographical distribution of radiotherapy centers in Belgium as well as its possible limitations.

Results-conclusion. - It appears that the current situation is on the average acceptable in terms of accessibility to the service and that the method presents huge potentialities for decision making so as to yield a spatial system that is both efficient and equitable.

@ 2012 Société française de radiothérapie oncologique (SFRO). Published by Elsevier Masson SAS. All rights reserved.

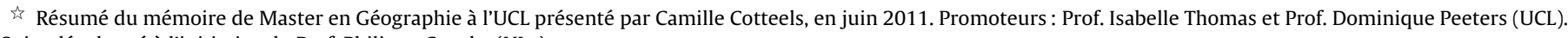
Sujet développé à l'initiative du Prof. Philippe Coucke (ULg).

$\diamond$ Summary of Master thesis in Geography at UCL presented by Camille Cotteels, in June 2011. Promoters: Prof. Isabelle Thomas and Prof. Dominique Peeters (UCL).

Subject developed at the initiative of Prof. Philippe Coucke (ULg).

* Auteur correspondant.

Adresse e-mail : isabelle.thomas@uclouvain.be (I. Thomas).
} 


\section{Introduction}

En moyenne, on estime que $60 \%$ des personnes atteintes d'un cancer reçoivent une radiothérapie à un moment ou un autre de leur traitement. Ce traitement se pratique dans un centre spécialisé, le plus souvent en ambulatoire, à des rythmes variables selon les traitements, mais pouvant être journaliers pendant cinq à huit semaines. Les séances ne durent que quelques minutes mais nécessitent le déplacement du patient. L'accessibilité spatiale de tels centres est donc essentielle mais souvent ignorée dans les analyses coûts-bénéfices. Ce déplacement engendre en effet un coût de transport non négligeable pour la société, l'environnement et le malade lui-même, et il se mesure en distance, temps, euros, émissions de $\mathrm{CO}_{2}$ et fatigue. Il a déjà été démontré que les patients sont généralement prêts à parcourir une distance au plus de $35 \mathrm{~km}$ en Europe [1,2]. Cette distance est un facteur qui peut non seulement entraver la compliance du traitement et, par ce biais, son efficacité [3], mais aussi fortement influencer le recours à ce type de traitement (l'éloignement engendre le non-usage - [4]). Nous nous sommes intéressés uniquement à la distance kilométrique sans nous attarder sur les coûts monétaires, les files d'attente, etc.

Nous proposons ici un modèle simple de localisation des centres de façon à minimiser la distance totale parcourue par les patients se rendant au service. Nous avons considéré que tous les centres de radiothérapie offraient le même service tout en restant conscients que la réalité est bien plus complexe. La question de la dotation de chacun des centres retenus en matériel et en ressources humaines est d'une autre nature et exige le recours à des informations sur le fonctionnement du système dont nous ne disposions malheureusement pas. La disponibilité des données, le temps qui nous était imparti et les nombreux facteurs qui peuvent entrer en compte nous ont limité à une étude simple : cartographier les localisations des centres de radiothérapie en 2011 et évaluer les disparités spatiales observées par des outils géographiques appropriés. Toutefois, la simplification des inputs ne nous permet pas de tirer des conclusions pratiques à proposer aux structures sanitaires dans l'immédiat. L'étude révèle cependant l'importance relative des centres retenus, donc des lieux où du matériel plus sophistiqué pourrait être installé, de même que les problèmes d'accès des patients aux centres. Cet article présente la méthodologie et les résultats d'une étude préliminaire.

\section{Matériel et méthodes}

\subsection{Localisations en 2011}

À notre grande surprise, il a été difficile d'obtenir en 2011 une vue instantanée de l'ensemble des implantations des centres de radiothérapie à l'échelle d'un pays comme la Belgique, sans parler des "aires de chalandise " ${ }^{1}$. En ce qui concerne la localisation des centres, la collecte des données a été faite par enquêtes spécifiques auprès de différentes instances et administrations, et validée auprès d'acteurs dans le domaine. L'objectif était simplement de connaître la localisation des centres de radiothérapie et quelquesunes de leurs caractéristiques. Nous entendons par centre de radiothérapie tout endroit qui pratique la radiothérapie, quelle qu'elle soit.

Pour rappel, en Belgique, on estime qu'environ un homme sur trois et une femme sur quatre seront atteints d'un cancer avant leur $75^{\mathrm{e}}$ année. Sachant que la Belgique compte 11 millions d'habitants

\footnotetext{
1 Aires dans lesquelles se recrutent les patients, aires de provenance des patients.
}

sur une superficie de $30528 \mathrm{~km}^{2}$, le problème de transport vers le lieu de soin prend tout son sens. Le problème devient d'autant plus aigu lorsqu'on sait que le Pays est divisé en trois régions administratives (région Flamande, région Wallonne et région de Bruxelles-Capitale) et que la région de Bruxelles-Capitale est essentiellement urbaine. Rappelons aussi que Bruxelles est la capitale mais que l'agglomération s'étend bien au-delà des limites de la région [5] et que les deux autres régions sont caractérisées par une histoire et une géographie différentes [6]. Le nord du pays est plus densément peuplé et caractérisé par un maillage urbain ancien et dense alors que le Sud a fortement été marqué par l'industrialisation du $\mathrm{XIX}^{\mathrm{e}}$ siècle et est caractérisé par un maillage urbain lâche, un relief marqué et donc des distances à parcourir plus grandes [7].

La Belgique comptait, en 2011, 36 centres de radiothérapie répartis entre 27 communes différentes (Fig. 1). La répartition suivait globalement la hiérarchie urbaine ${ }^{2}$ avec une forte concentration dans les grandes villes.

Le Tableau 1 montre la relation qui existait entre le nombre de centres de radiothérapie par agglomération ou commune et la place de celle-ci dans la hiérarchie urbaine. Cette relation était aussi évoquée dans [8], qui traitait de l'offre hospitalière (nombre de lits et diversité offerte). Nous avons choisi de regrouper les communes en agglomérations car les grandes villes s'étalent sur les communes voisines. Regardons également l'importance de l'agglomération de Bruxelles (ville primatiale) et au fait qu'elle abrite trois facultés de médecine et donc trois hôpitaux universitaires.

Pour chaque centre de radiothérapie, les caractéristiques suivantes ont également été obtenues auprès du Service public fédéral santé publique pour l'année 2011 : l'existence de centres mères et de centres antennes spatialement différenciées ${ }^{3}$ (Fig. 2), le type d'hôpital qui hébergeait le centre (général, général à caractère universitaire ou universitaire) (Fig. 3) et l'appartenance à un réseau (publics ou privés) (Fig. 4).

Les caractéristiques des hôpitaux abritant un centre de radiothérapie ou celles du centre lui-même ont engendré quelques surprises spatiales : conformément à l'attente, lorsqu'une commune, et a fortiori une agglomération urbaine, disposait de plusieurs centres de radiothérapie, les caractéristiques de ces derniers étaient souvent hétérogènes. C'est ainsi que, par exemple, La Louvière possédait deux centres: l'un était un centre "mère" dans un hôpital dit général, l'autre était un "centre antenne" dans un hôpital général à caractère universitaire, tous deux distants de moins d'un kilomètre. Ces «doublons" pouvaient être à l'origine d'une offre pléthorique par rapport aux besoins de la population locale mais aussi d'une dispersion des moyens conduisant à terme à des questions d'efficacité. Le surcoût engendré par l'existence de types différents de centres et de réseaux concurrents relève d'un autre débat que nous n'entamerons pas ici. Nous nous sommes limités aux localisations.

Simple outil cartographique, la Fig. 5 trace autour de chaque centre existant un cercle de distance croissante par "pas" de $10 \mathrm{~km}$ (distance à vol d'oiseau, dite euclidienne). Il s'agit certes d'une approximation grossière mais qui, pour un réseau dense, n'est pas trop exagérée [7]. Les centres se localisaient dans des

\footnotetext{
2 Classement des villes les unes par rapport aux autres, à partir de critères reposant sur l'importance de la population, les services et les équipements offerts par la ville, ses fonctions administrative, économique, culturelle, universitaire, etc. La hiérarchie distingue un certain nombre de classes: "grande ville», "ville régionale", "petite ville" et "commune non urbaine". Les petites villes sont divisées en trois sousclasses suivant leur niveau d'équipement (bon, moyen et faible) tout comme les communes non urbaines.

3 Une loi belge de 2002 limite le nombre de centres à 25. Pour contrer cette limite, certains hôpitaux " mères » ont créé des antennes, possible depuis une loi de 2005 , ce qui fait que le nombre de centres est passé à 36 .
} 


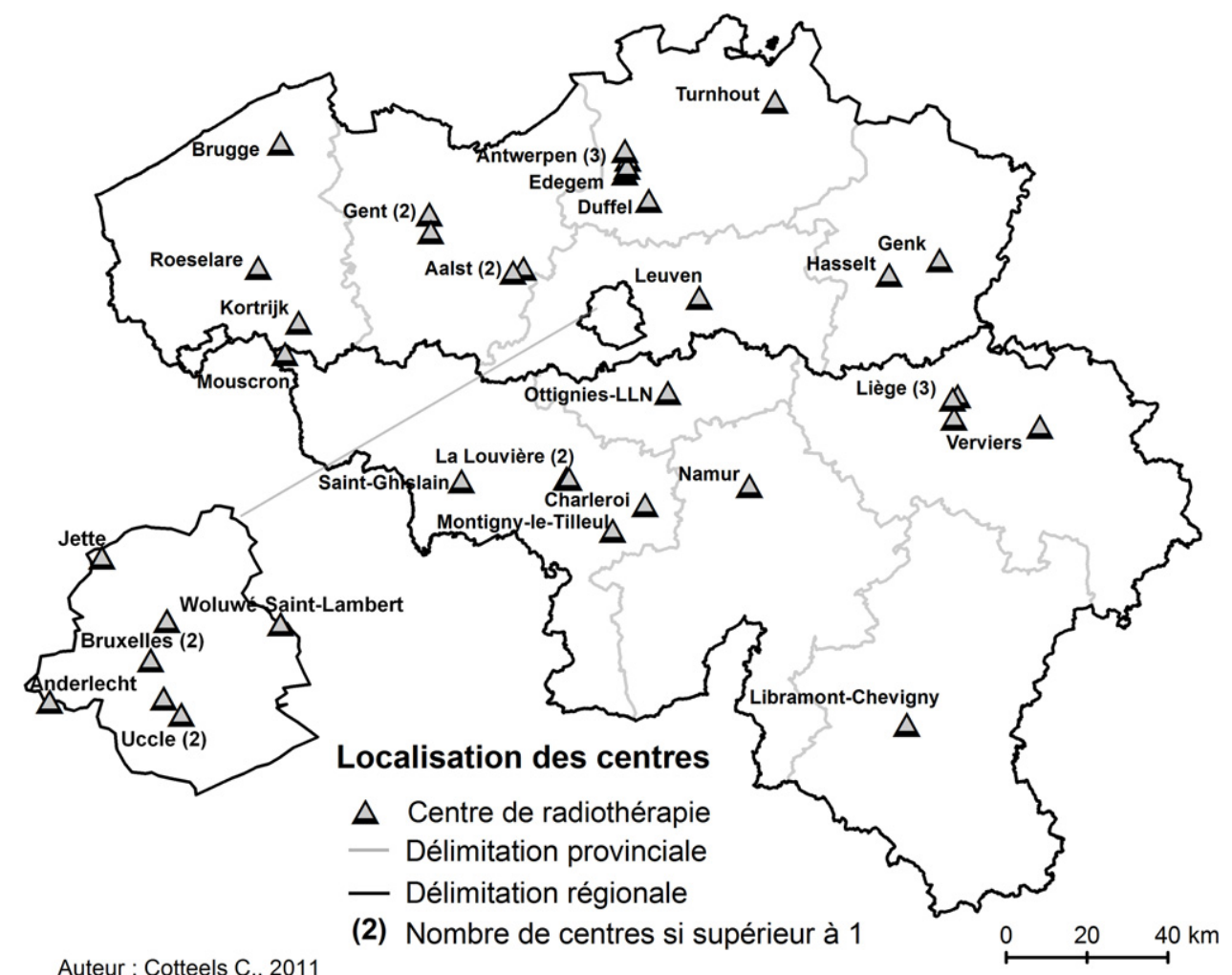

Fig. 1. Localisation des centres de radiothérapie en Belgique en 2011.

Location of radiotherapy centers in Belgium in 2011.

Récolte personnelle C. Cotteels, 2011.

Tableau 1

Relation entre le nombre de centre de radiothérapie par agglomération et la place de la ville ou commune dans la hiérarchie urbaine.

Relationship between the number of radiotherapy center in town and the place of the city or municipality in the urban hierarchy.

\begin{tabular}{|c|c|c|c|c|c|}
\hline Commune & Nombre de centres & Agglomération & Hiérarchie urbaine [9] & $\begin{array}{l}\text { Nombre de } \\
\text { centres }\end{array}$ & $\begin{array}{l}\text { Nombre d'habitants } \\
{[10]}\end{array}$ \\
\hline Bruxelles & 2 & \multirow[t]{5}{*}{ Bruxelles } & \multirow{5}{*}{$\begin{array}{l}\text { Grande } \\
\text { ville }\end{array}$} & \multirow[t]{5}{*}{7} & \multirow[t]{5}{*}{1474234} \\
\hline Jette & 1 & & & & \\
\hline Anderlecht & 1 & & & & \\
\hline Woluwe-Saint-Lambert & 1 & & & & \\
\hline Uccle & 2 & & & & \\
\hline Antwerpen & 3 & \multirow[t]{2}{*}{ Antwerpen } & \multirow{2}{*}{$\begin{array}{l}\text { Grande } \\
\text { ville }\end{array}$} & \multirow[t]{2}{*}{4} & \multirow[t]{2}{*}{721818} \\
\hline Edegem & 1 & & & & \\
\hline Liège & 3 & Liège & Grande ville & 3 & 482310 \\
\hline Charleroi & 1 & \multirow[t]{2}{*}{ Charleroi } & \multirow{2}{*}{$\begin{array}{l}\text { Grande } \\
\text { ville }\end{array}$} & \multirow[t]{2}{*}{2} & \multirow[t]{2}{*}{289357} \\
\hline Montigny-le-Tilleul & 1 & & & & \\
\hline Gent & 2 & Gent & Grande ville & 2 & 281406 \\
\hline Brugge & 1 & - & Ville régionale & 1 & 116969 \\
\hline Leuven & 1 & - & Ville régionale & 1 & 114092 \\
\hline Kortrijk & 1 & - & Ville régionale & 1 & 113478 \\
\hline Namur & 1 & - & Ville régionale & 1 & 108514 \\
\hline Genk & 1 & - & Ville régionale & 1 & 82656 \\
\hline Verviers & 1 & - & Ville régionale & 1 & 79131 \\
\hline Aalst & 2 & - & Ville régionale & 2 & 79052 \\
\hline La Louvière & 2 & - & Ville régionale & 2 & 77726 \\
\hline Hasselt & 1 & - & Ville régionale & 1 & 72315 \\
\hline Roeselare & 1 & - & Ville régionale & 1 & 56893 \\
\hline Turnhout & 1 & - & Ville régionale & 1 & 40441 \\
\hline Mouscron & 1 & - & Petite ville & 1 & 53174 \\
\hline Ottignies-Louvain-la-Neuve & 1 & - & Petite ville & 1 & 30005 \\
\hline Saint-Ghislain & 1 & - & Petite ville & 1 & 22844 \\
\hline Libramont-Chevigny & 1 & - & Petite ville & 1 & 10230 \\
\hline Duffel & 1 & - & Commune non urbaine & 1 & 16140 \\
\hline
\end{tabular}




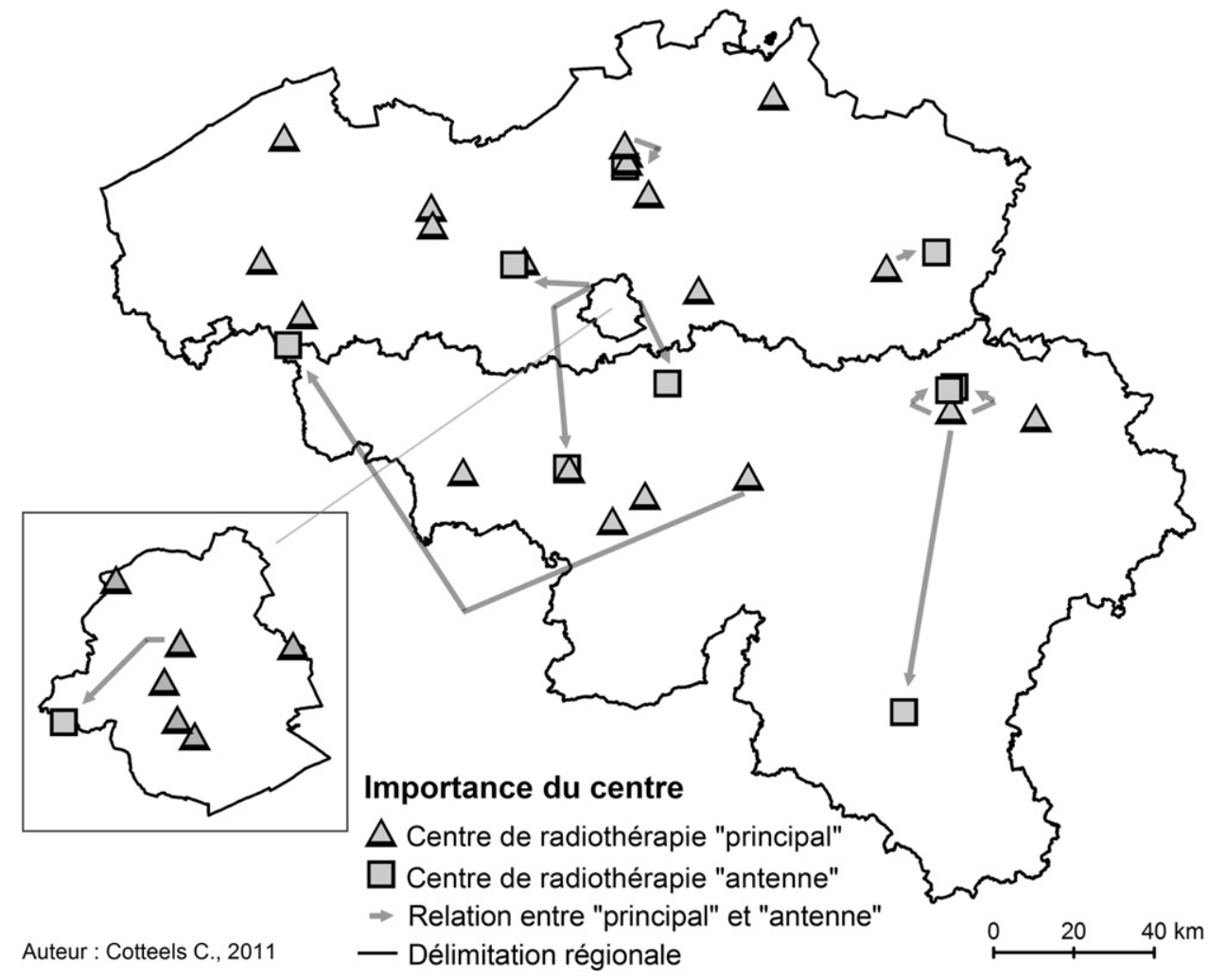

Fig. 2. Types de centre de radiothérapie en Belgique en 2011 : centre mère ou principal et antenne. Types of radiotherapy center in Belgium in 2011: principal center and antenna. Récolte personnelle C. Cotteels, 2011.

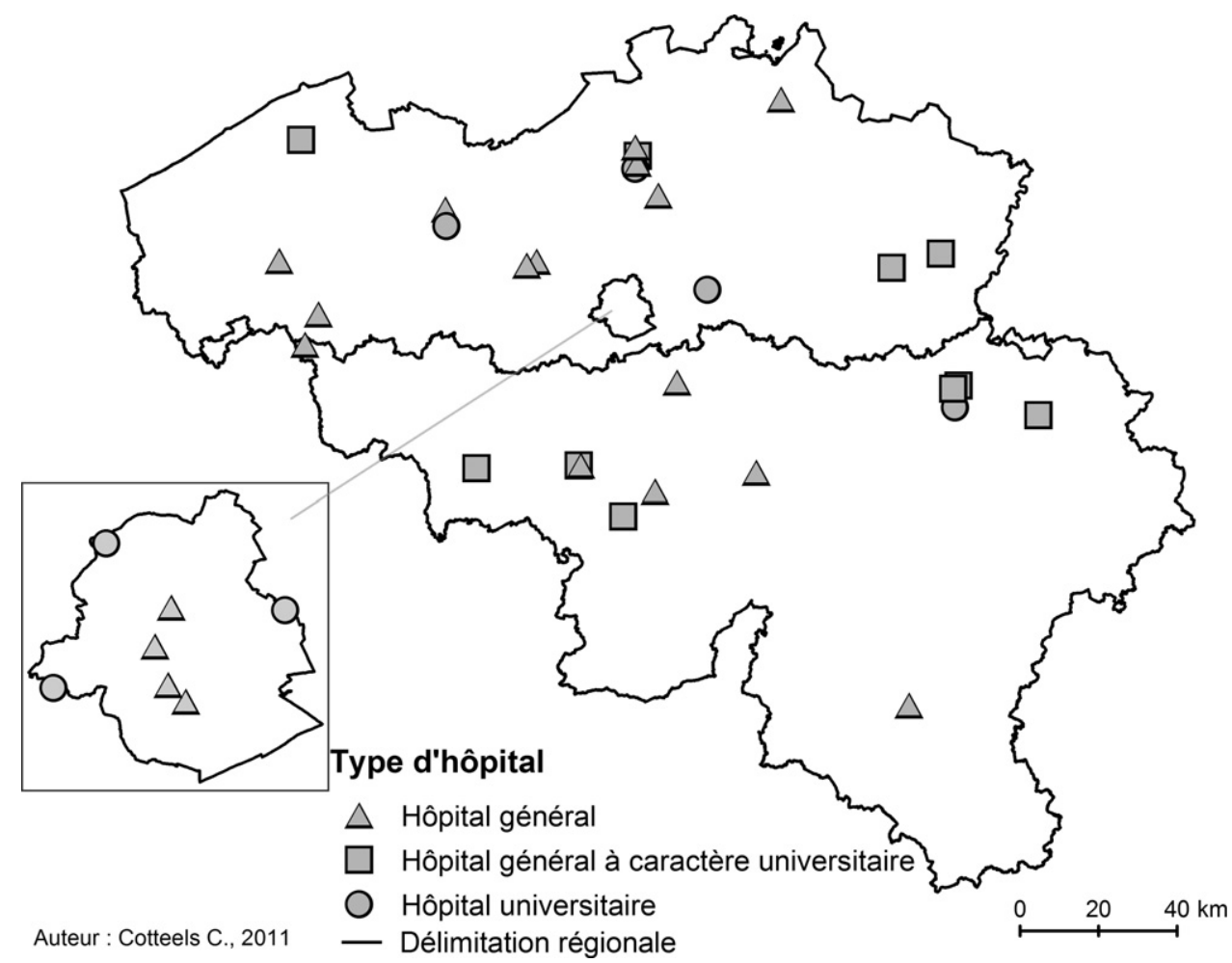

Fig. 3. Centres de radiothérapie en Belgique en 2011 selon le type d'hôpital : universitaire, à caractère universitaire ou général. Radiotherapy centers in Belgium in 2011 according to the type of hospital: academic, general of an academic, general. Récolte personnelle C. Cotteels, 2011. 


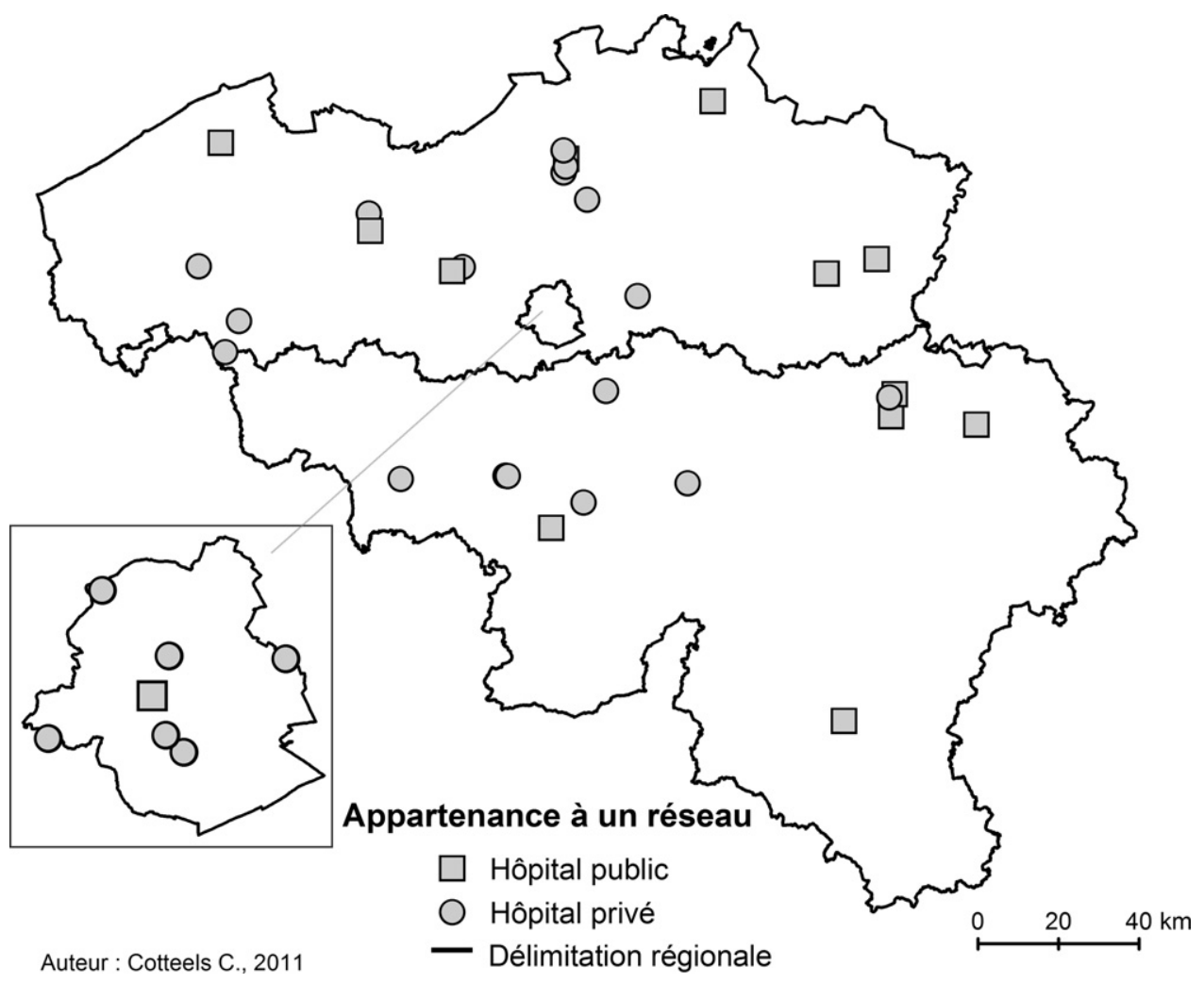

Fig. 4. Centres de radiothérapie en Belgique en 2011 selon le caractère privé ou public de l'hôpital. Radiotherapy centers in Belgium in 2011 according to the private or public hospitals. Récolte personnelle C. Cotteels, 2011.

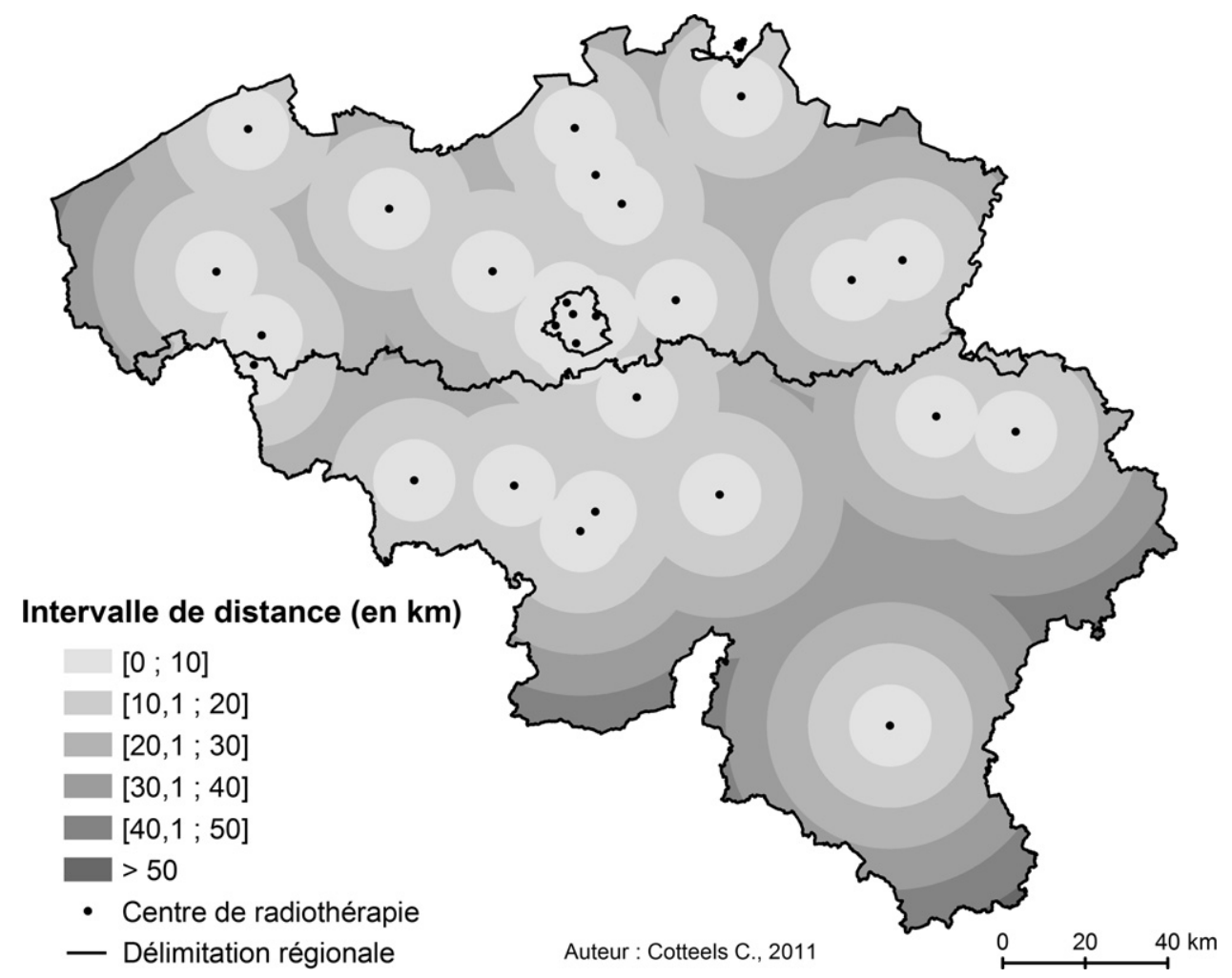

Fig. 5. Intervalle de distance à vol d'oiseau autour des centres de radiothérapie actuels, par pas de $10 \mathrm{~km}$. Range of distance in a straight line around the existing radiotherapy centers in steps of $10 \mathrm{~km}$. 
Tableau 2

Pourcentages des communes et de la population situées à un certain intervalle de distance d'un centre de radiothérapie.

Percentages of municipalities and population located in a certain interval distance of a radiotherapy center.

\begin{tabular}{lcc}
\hline Intervalle de distance $($ en $\mathrm{km})$ & $\begin{array}{l}\text { Pourcentage des } \\
\text { communes }\end{array}$ & $\begin{array}{l}\text { Pourcentage de la } \\
\text { population }\end{array}$ \\
\hline$[0-10]$ & 34,5 & 55,2 \\
{$[10,1-20]$} & 38,5 & 30,0 \\
{$[20,1-30]$} & 19,5 & 11,7 \\
{$[30,1-40]$} & 7,6 & 2,3 \\
{$[40,1-50]$} & 1,9 & 0,9 \\
Total & 100 & 100 \\
\hline
\end{tabular}

communes fortement peuplées, plus de $50 \%$ de la population belge se trouvaient à moins de $10 \mathrm{~km}$ d'un centre alors que cela représentait près de $35 \%$ des communes (Tableau 2). Conformément à l'attente, on constatait qu'une partie du territoire était distante de plus de $35 \mathrm{~km}$ d'un centre, soit près de $4 \%$ des communes et près de $2 \%$ de la population. Les zones peu peuplées et souvent périphériques étaient les plus inaccessibles : les patients qui y résidaient avaient un accès plus difficile aux centres de radiothérapie avec les conséquences que l'on connaît. Les centres de radiothérapie étrangers et localisés près de la frontière n'ont pas été pris en compte.

\subsection{Modèle de localisation optimale}

Un modèle de recherche opérationnelle ( $p$-médiane) a été appliqué pour déterminer l'organisation spatiale optimale des centres de radiothérapie, c'est-à-dire les localisations les plus favorables. Ce modèle avait l'avantage d'être facilement implémenté et de rechercher un compromis entre l'efficacité, exprimée par la minimisation de la distance totale entre patients et centres, et l'équité, exprimée par la distance que doit parcourir l'utilisateur le plus défavorisé [11-14]. En comparant cette situation optimale à la situation décrite au point précédent, il était possible de s'interroger sur certaines localisations, voire de suggérer des relocalisations ou de nouvelles localisations. La formulation utilisée est très simple et essentiellement spatiale. Cette démarche est toutefois relativement réaliste au vu d'applications antérieures pour d'autres services publics $[15,16]$.

En d'autres termes, le modèle de la $p$-médiane minimise la distance totale parcourue par les patients (=la demande) vers les centres (= l'offre) sous un ensemble de contraintes. Ce modèle s'est souvent avéré très utile pour la localisation optimale de services publics, même avec des variables peu nombreuses [11-13,16-18]. Les outputs de la $p$-médiane sont la localisation des centres sélectionnés, l'affectation de la clientèle à ces centres, la forme des zones desservies, l'accessibilité totale du système, la distance moyenne parcourue par un usager et la distance séparant l'usager le plus mal desservi du site auquel il est affecté. Ainsi grâce à ce modèle, il était possible de dire quel était le nombre idéal de centres de radiothérapie qui permettait de desservir toute la Belgique si on minimisait la distance, quels seraient les centres à supprimer tout en maintenant un niveau d'efficacité et d'équité raisonnables ou au contraire quelles étaient les régions mal desservies, que faire pour y remédier et à quel investissement faut-il consentir.

Le modèle de la $p$-médiane se formule comme suit. La région étudiée est représentée par un ensemble de points indicés $i=1, \ldots$, $I$ et $a_{i}$ est la demande émanant de $i$. Soit $j=1, \ldots ., J$ un ensemble de sites où on peut éventuellement installer des centres. $d_{i j}$ désigne la distance entre les lieux $i$ et $j$. La variable $y_{j}$ prend la valeur 1 si on implante un centre en $j$ et 0 sinon. La variable $x_{i j}$ vaut 1 si la demande en $i$ est satisfaite par un centre en $j$ et 0 sinon. La fonction objectif
Tableau 3

Hypothèses émises pour estimer la demande.

Assumptions to estimate demand.

\begin{tabular}{ll}
\hline & Hypothèses \\
\hline Hypothèse 1 & $\begin{array}{l}\text { Tout habitant est un candidat potentiel à la radiothérapie ; } \\
\text { dans ce cas la population totale au } 1^{\text {er }} \text { janvier 2007 a été } \\
\text { utilisée }\end{array}$ \\
Hypothèse 2 & $\begin{array}{l}\text { Seules les personnes âgées de } 60 \text { ans ou plus sont } \\
\text { candidates potentielles à la radiothérapie }\end{array}$ \\
Hypothèse 3 & $\begin{array}{l}\text { Tous les habitants sont susceptibles d'avoir recours à la } \\
\text { radiothérapie mais la probabilité d'y avoir recours } \\
\text { augmente avec l'âge suivant la formule brute : } \\
n_{i}=b_{1} \text { (nombre de personnes de }>60 \text { ans) }+b_{2} \text { (nombre de } \\
\text { personnes de }<60 \text { ans) } \\
\text { Où } b_{1} \text { et } b_{2} \text { valent respectivement } 0,3 \text { et } 0,7 \text { et sont } \\
\text { inspirées du Belgian Cancer Registry (en 2011) }\end{array}$ \\
\hline
\end{tabular}

Tableau 4

Hypothèses émises pour estimer l'offre.

Assumptions to estimate supply.

\begin{tabular}{ll}
\hline & Hypothèses \\
\hline Hypothèse 1 & $\begin{array}{l}n_{1}=589 \text {. Dans ce cas, les } 589 \text { communes de Belgique sont } \\
\text { potentiellement susceptibles d'accueillir un centre de } \\
\text { radiothérapie }\end{array}$ \\
& $\begin{array}{l}n_{2}=122 . \text { Ici, les centres de radiothérapie ne seraient } \\
\text { installés que dans des communes disposant déjà d'au } \\
\text { mypoins un hôpital général }\end{array}$ \\
& $\begin{array}{l}n_{3}=27 . \text { Enfin, dans ce cas les points d'offre potentiels } \\
\text { correspondent aux 27 communes possédant aujourd'hui } \\
\text { Hypothèse } 3 \\
\end{array}$ \\
& $\begin{array}{l}\text { fonctionnel. Dans ce cas, nous pouvons estimer } \\
\text { l'affectation optimale des centres actuels mais aussi } \\
\text { proposer éventuellement des suppressions/fermetures }\end{array}$ \\
\hline
\end{tabular}

C est la distance totale parcourue par les utilisateurs du service se rendant au centre auquel ils sont affectés :

$C^{*}=\operatorname{Min} C(y, X)=\sum_{i=1}^{I} \sum_{j=1}^{J} a_{i} d_{i j} x_{i j}$

Les contraintes du problème sont les suivantes :

$\Sigma_{j=1}^{J} x_{i j}=1, i=1, \ldots, I$

Cette contrainte garantit que toute la demande pour le service soit satisfaite. Chaque point de demande est affecté à un et un seul site d'offre. Il en résulte que les aires de services des unités implantées ne se recouvrent pas.

$x_{i j} \leq y_{j}, i=1, \ldots, I ; j=1, \ldots, J$

La demande en $i$ ne peut être satisfaite par le site $j$ que si une unité y est implantée.

$\Sigma_{j=1}^{J} y_{j}=p$

où $p$ est le nombre de centres à localiser.

Ce problème a été résolu au moyen du programme FIXMED, basé sur un algorithme développé dans [17]. En revanche, la cartographie des résultats a été réalisée avec ArcGIS.

Le niveau d'agrégation des données retenu était la commune ( $n=589$ ). Plusieurs hypothèses de travail (simples et brutes) ont été faites afin d'évaluer la demande (Tableau 3). Elle reposait donc uniquement sur des données de population communale. Les communes potentiellement susceptibles d'accueillir un centre de radiothérapie constituaient l'offre. Plusieurs définitions des ensembles de lieux potentiels d'offre ont été imaginés afin d'évaluer la robustesse des résultats (Tableau 4). Nous avons supposé dans notre modèle que tous les centres pratiquaient des radiothérapies 

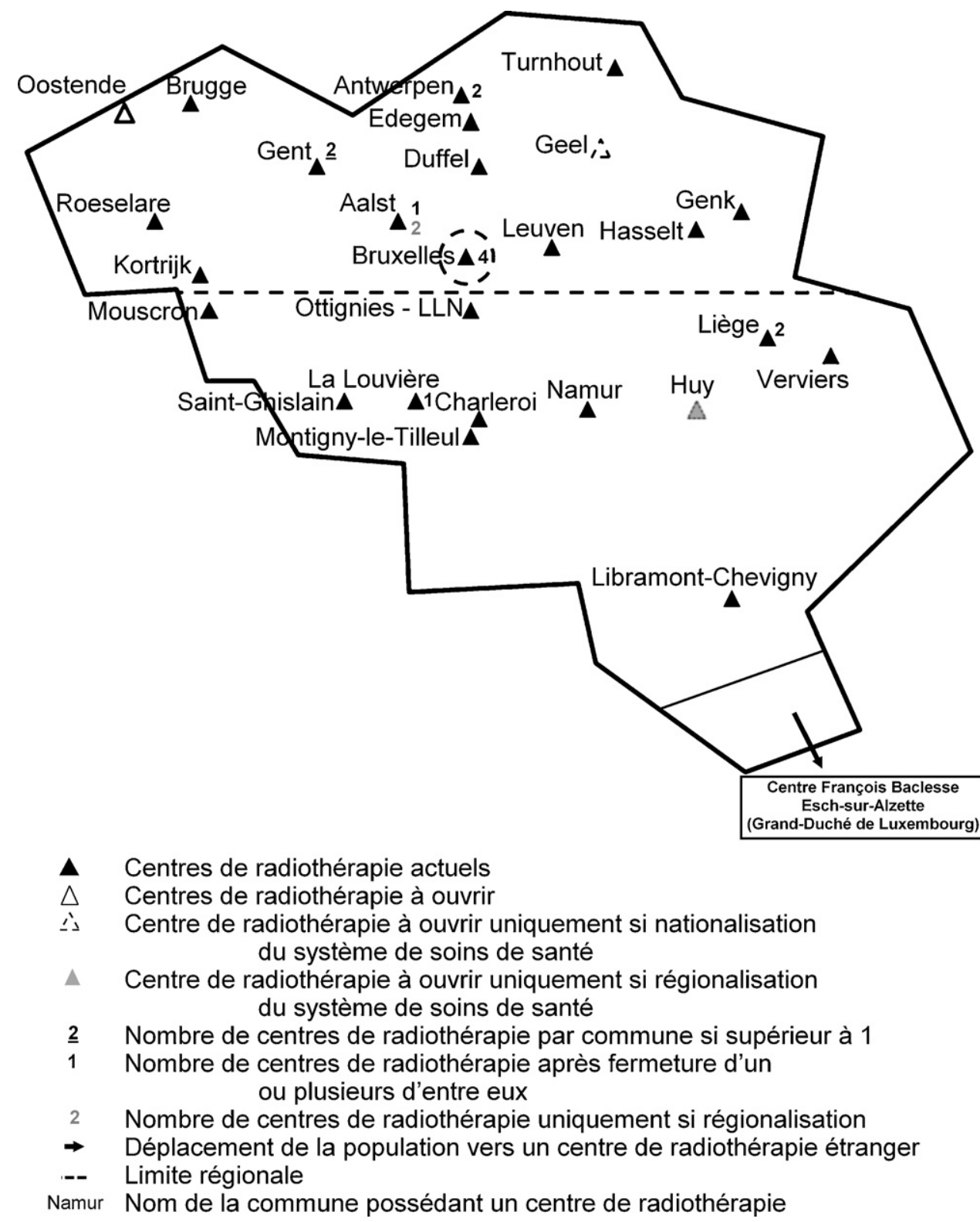

Fig. 6. Localisations jugées préférentielles et comparaison avec la situation actuelle. Considered preferential locations and compared with the current situation.

identiques sans prendre en compte la dotation technique ou le personnel affecté. La mesure de $d_{i j}$ est la distance à vol d'oiseau.

Plusieurs séries de simulations ont été réalisées en faisant varier les conditions d'offre et de demande. Il apparaissait que même en changeant la définition de l'offre et/ou la demande, l'optimalité des localisations des centres de radiothérapie variait peu. Les résultats étaient donc peu sensibles aux définitions de la demande et de l'offre: une certaine robustesse des résultats était observée. L'espace serait donc fortement structurant confirmant ainsi [19]. Dans un second temps, les localisations optimales des centres ont été calculées séparément pour la Wallonie et la Flandre. Pour chacune des deux régions, nous avons ajouté la région de Bruxelles-Capitale et nous avons regardé quelle(s) commune(s) était/étaient affectée(s) aux centres de radiothérapie bruxellois. Cette simulation nous permettait de vérifier l'effet de la perméabilité de la frontière linguistique mais aussi de mesurer le coût d'une éventuelle régionalisation dans le domaine.

\section{Résultats}

La Fig. 6 synthétise l'ensemble des résultats obtenus. En moyenne, les localisations jugées préférentielles étaient proches de la configuration de 2011. Parmi les localisations suggérées par le modèle, deux nouvelles localisations semblaient s'imposer: Oostende et Geel. Il aurait également été nécessaire d'ouvrir un centre à Arlon, mais vu le petit nombre de patients potentiels affectés à ce centre, nous ne l'avons pas proposé. De plus, ce centre aurait été proche du centre d'Esch-sur-Alzette (Grand-Duché du Luxembourg) avec lequel la Belgique a des accords transfrontaliers (INTERREG) pour la radiothérapie. Nos simulations suggéraient de supprimer des centres à La Louvière, Aalst, Bruxelles, Liège et Antwerpen. Ce résultat a cependant été pris avec réserve étant donné les conditions d'application simples, voire simplistes, du modèle. Malgré tout, ces résultats nous poussent à réfléchir sur la multiplicité des centres dans les grandes agglomérations due à leurs caractéristiques spécifiques. 
En considérant la Flandre et la Wallonie séparément, les localisations et relocalisations proposées étaient similaires à celles obtenues pour l'ensemble du pays, à l'exception de l'ouverture d'un site à Huy au lieu d'un site à Geel. En cas de régionalisation du système de soins, nous avions émis l'hypothèse que Bruxelles restait accessible aux deux autres régions, seul le « passage » de la frontière entre les deux régions serait rendu impossible.

Sept centres étaient présents en 2011 en Région de BruxellesCapitale, le modèle suggérait de réduire ce nombre. Toutefois, rappelons que Bruxelles, comme de très nombreuses villes dans le monde, connaît une forte périurbanisation. Une des particularités de Bruxelles est qu'elle s'étale, au-delà des limites de la Région de Bruxelles-Capitale en Flandre et en Wallonie, posant ainsi de nombreux problèmes de gestion urbaine et communale [5] qui s'avéraient dans notre cas importants. De plus, comme toute grande ville, elle attire des fonctions supérieures et donc aussi médicales [6]. La réduction proposée devait donc être envisagée avec prudence vu la position de Bruxelles dans la hiérarchie urbaine.

Au cours de notre étude, nous avons appris qu'un centre allait fermer ses portes à Antwerpen et que deux antennes allaient ouvrir (l'une à Sint Niklaas et l'autre à Brasschaat). Ces changements n'avaient pas été pris en considération et ne semblaient pas s'inscrire dans la lignée des résultats de nos modèles, en tous cas à l'échelle d'analyse adoptée ici (communes). À l'échelle d'une ville et de sa banlieue, c'est une autre histoire qui pourrait être analysée avec les mêmes outils mais à une échelle spatiale plus fine (tels les secteurs statistiques).

Une régionalisation des soins de santé générera un surcoût aux régions. En effet, l'aire théorique d'affectation de certains centres était à cheval sur la frontière linguistique. Une partie de la population était donc affectée à un centre situé dans une région différente de celle où elle résidait. Avec la régionalisation des soins de santé et les règles spatiales très strictes que nous avons adoptées, le modèle suggérait de localiser plus de centres, de petite taille, afin de veiller à ce que les patients ne parcourent pas une longue distance (pour rappel la $p$-médiane cherche un compromis entre efficacité et équité spatiales ; dans la version présentée aucune masse critique de taille n'a été imposée).

Pour différentes raisons, les centres de radiothérapie se sont multipliés dans notre pays; cette croissance de l'offre était entre autre liée, à la présence de plusieurs réseaux d'établissements de soins de santé ou à la volonté de faire croître le pôle santé d'une commune ou d'un établissement par la création d'une ou plusieurs antennes. Le nombre de centres se multipliait alors que le nombre d'habitants n'augmentait pas ou peu. Et pendant ce temps, certaines communes restaient mal desservies. La localisation de centres de radiothérapie devrait urgemment être considérée dans son ensemble comme un réseau et non un ensemble de localisations indépendantes afin de couvrir le pays efficacement ET équitablement. Pour rappel, le but de chaque centre est et reste de soigner la population.

\section{Discussion}

Nous n'avons pas pu comparer nos résultats à une autre étude car à notre connaissance, c'était la première fois qu'une telle étude spatiale était réalisée pour la radiothérapie. Le modèle proposé ici peut paraître caricatural ; la situation est simplifiée à outrance mais nous restons convaincus de la validité des résultats et du message donné. Certes, de nombreuses pistes peuvent maintenant être poursuivies en affinant les données de l'offre, de la demande ou de la distance. Toutefois - par définition - un modèle consiste à créer une représentation simplifiée.

Ainsi pour l'offre, nous aurions pu tenir compte du type d'hôpital (universitaire, à caractère universitaire ou général), du type de centre ("mère " ou "antenne »), des spécificités de chaque centre (spécialisation, matériel, nombre de médecins et leur spécialisation, files d'attente, réputation, etc.).

Pour la demande, les différences spatiales dans la probabilité de développer un cancer et de suivre une radiothérapie auraient pu être prises en compte de même que la distinction suivant le sexe des patients potentiels, la structure d'âge de la population, la nature de l'affection, etc.

La distance est une mesure de l'accessibilité des patients au service. Nous avons opté ici pour la distance à vol d'oiseau. Nous pourrions ultérieurement poursuivre l'analyse avec des évaluations sensées plus réalistes comme les distances ou les temps de déplacement mesurés sur les réseaux de transport existants. Cependant, tout porte à croire que les écarts seraient minimes en moyenne à l'échelle de la Belgique, sauf peut-être si l'on prenait en compte des effets de la congestion qui ne sont pas négligeables localement. Nous aurions pu également utiliser les coûts de transport directs et indirects. En Belgique, ceux-ci sont partiellement remboursés aux patients, ce qui justifie du point de vue social qu'on cherche à les minimiser.

Dans l'implémentation de notre modèle, nous avons imposé certaines contraintes de base mais nous aurions pu en mettre d'avantage. Nous aurions, par exemple, pu appliquer une contrainte de capacité en imposant - par exemple - des nombres minimaux et maximaux de patients affectés, voire d'autres contraintes sur le budget disponible ou sur le nombre de personnes employées. Nous pourrions aussi affiner par rapport à la dilution de l'expertise et le risque de voire la technicité de traitement se réduire en multipliant le nombre de structures (compte tenu des coûts d'investissement et de maintenance des plateaux techniques High Tech). Le modèle ne pourra en revanche pas tenir compte des imprévus : panne et temps de maintenance du matériel ou des jours fériés. De même le temps de traitement sera calculé à partir d'une moyenne. Il est impossible de traiter les données de manière individuelle. Ainsi, par exemple, le nombre de patients par machine par jour sera approximé à partir du temps moyen d'une séance de radiothérapie (comprenant la séance et le temps de réglage de l'équipement).

Pour incorporer les multiples complexifications, nous avons besoin d'une base de données fiable, exhaustive et étendue à tout le pays, or celle-ci n'existe pas. Cette base de données devrait contenir des informations sur l'ensemble des centres de radiothérapie en activité, des données relatives à l'état de l'ensemble des équipements dédiées à la radiothérapie, à l'activité de chaque établissement et aux effectifs médicaux et paramédicaux. Cette base de données pourrait s'inspirer de l'enquête annuelle réalisée en France par la Société française de radiothérapie oncologique (SFRO).

Ces quelques éléments rappellent les limites de l'approche mais également les potentialités du modèle utilisé. Nous croyons toutefois, que malgré ces nombreuses limites, la cartographie (de 2011 et jugée préférentielle) des centres de radiothérapie en Belgique proposée ici constitue une première piste intéressante de réflexion "géo-politique», le point de départ d'une réflexion sur l'investissement et la gestion publique.

\section{Conclusion}

Cette étude ne constituait qu'un travail préliminaire à une éventuelle analyse plus approfondie. Notre travail consistait simplement à cartographier la localisation des centres de radiothérapie, à évaluer cette situation et à proposer éventuellement des solutions pour améliorer la couverture spatiale de 2011 en Belgique. Nous avons utilisé différents outils exploratoires simples afin d'y parvenir. Les résultats obtenus donnent une tendance, mais une tendance forte et, semble-t-il, robuste. Ils permettent d'orienter le débat entre décideurs et payeurs. Bien des pistes peuvent être envisagées 
pour poursuivre l'analyse et particulièrement affiner les résultats ; le modèle de localisation optimale mériterait entre autre d'être développé en incluant une estimation plus fine de la demande, une mesure plus réaliste de la distance des coûts de transport voire d'inclusion partielle ou totale de leur prise en charge, des coûts d'investissement et de fonctionnement, des problèmes de capacité et de listes d'attente, du cheminement spatial du patient, etc. Ces multiples pistes sortent du cadre de cet exercice mais laissent augurer une analyse spatiale passionnante, multidisciplinaire et multiéchelle, conduisant à la construction d'un outil d'aide à la décision performant.

\section{Déclaration d'intérêts}

Les auteurs déclarent ne pas avoir de conflits d'intérêts en relation avec cet article.

\section{Références}

[1] Pignon T, Paoli JB, Scalliet P. La radiothérapie du sujet âgé. In: Morère JF, Rainfray M, editors. Cancer du sujet âgé. Paris: Springer-Verlag; 2007. p. 75-88.

[2] Kalogirou S, Mostratos N. Geographical access to health: modeling population access to Greek public hospitals. In: Proceedings of the 7th Pan-Hellenic Geographical Congress. 2004. p. 431-7.

[3] Athas W, Adams-Cameron M, Hunt W, Amir-Fazli A, Rey C. Travel distance to radiation therapy and receipt of radiotherapy following breast-conserving surgery. J Natl Cancer Inst 1999;92:269-71.

[4] Guidry J, Aday L, Zhang D, Winn R. Transportation as a barrier to cancer treatment. Cancer Pract 1997;5:361-6.
[5] Dujardin C, Thomas I, Tulkens H. Quelles frontières pour Bruxelles ? Une mise à jour. Reflets et perspectives de la vie économique, 2; 2007. p. 155-76.

[6] Thisse JF, Thomas I. Bruxelles au sein de l'économie belge: un bilan. Regards Economiques 2010;80:18.

[7] Vandenbulcke G, Steenberghen T, Thomas I. Mapping accessibility in Belgium: a tool for land-use and transport planning? J Transp Geogr 2009;17: 39-59.

[8] Trotta M. Évolution (1979-2008) des services d'hospitalisation en Belgique. Quels liens avec la hiérarchie urbaine? Bull Soc Geogr Liege 2010:53.

[9] Van Hecke E. Actualisation de la hiérarchie urbaine en Belgique. Bulletin trimestriel du Crédit Communal 1998;3:45-76.

[10] SPF Économie, P.M.E., Classes moyennes et Énergie (2011). Population de droit par commune au 1 janvier 2007, Bruxelles, Belgique [Données en lignes statbel.fgov.be].

[11] Daskin M. Network and discrete location: models, algorithms and applications. Chichester: Wiley \& Sons; 1995.

[12] Peeters D, Thomas I. Localisation des services publics : de la théorie aux applications. In: Sanders L, editor. Les modèles en analyse spatiales. Collection IGAT. Paris: Hermès-Science publications; 2001. p. 105-27.

[13] Drezner Z, Hamacher H, editors. Facility location - Applications and theory. New York: Springer-Verlag; 2004.

[14] Murray A. Advances in location modeling: GIS linkages and contributions. ] Geogr Syst 2010;12:335-54.

[15] Beguin H, Thomas I, Vandenbussche D. Weight variations within a set of demand points and location allocation issues: a case study of public libraries. Environ Plann A 1992;24:1769-79.

[16] Querriau X, Kissiyar M, Peeters D, Thomas I. Localisation optimale d'unités de soins dans un pays en voie de développement : analyse de sensibilité. Cybergeo: Eur J Geogr 2004 [Document 281, mise en ligne le 24 août 2004].

[17] Hanjoul P, Peeters D. A comparison of two dual-based procedures for solving the $p$-median problem. Eur J Operations Res 1985;20:387-96.

[18] Chevalier P, Thomas I, Geraets D, Goetghebeur E, Janssens O, Peeters D, et al. Locating fire stations: an integrated approach for Belgium. Socio Econ Plan Sci 2012;46:172-82.

[19] Thomas I. Transportation network and the optimal location of human activities. A numerical geography approach. Cheltenham: Edward Elgar Publishing Ltd; 2002. 